\title{
A phylogenomic analysis of Escherichia coli / Shigella group: implications of genomic features associated with pathogenicity and ecological adaptation
}

\author{
Yan Zhang and Kui Lin*
}

\begin{abstract}
Background: The Escherichia coli species contains a variety of commensal and pathogenic strains, and its intraspecific diversity is extraordinarily high. With the availability of an increasing number of $E$. coli strain genomes, a more comprehensive concept of their evolutionary history and ecological adaptation can be developed using phylogenomic analyses. In this study, we constructed two types of whole-genome phylogenies based on 34 E. coli strains using collinear genomic segments. The first phylogeny was based on the concatenated collinear regions shared by all of the studied genomes, and the second phylogeny was based on the variable collinear regions that are absent from at least one genome. Intuitively, the first phylogeny is likely to reveal the lineal evolutionary history among these strains (i.e., an evolutionary phylogeny), whereas the latter phylogeny is likely to reflect the wholegenome similarities of extant strains (i.e., a similarity phylogeny).

Results: Within the evolutionary phylogeny, the strains were clustered in accordance with known phylogenetic groups and phenotypes. When comparing evolutionary and similarity phylogenies, a concept emerges that Shigella may have originated from at least three distinct ancestors and evolved into a single clade. By scrutinizing the properties that are shared amongst Shigella strains but missing in other E. coli genomes, we found that the common regions of the Shigella genomes were mainly influenced by mobile genetic elements, implying that they may have experienced convergent evolution via horizontal gene transfer. Based on an inspection of certain key branches of interest, we identified several collinear regions that may be associated with the pathogenicity of specific strains. Moreover, by examining the annotated genes within these regions, further detailed evidence associated with pathogenicity was revealed.
\end{abstract}

Conclusions: Collinear regions are reliable genomic features used for phylogenomic analysis among closely related genomes while linking the genomic diversity with phenotypic differences in a meaningful way. The pathogenicity of a strain may be associated with both the arrival of virulence factors and the modification of genomes via mutations. Such phylogenomic studies that compare collinear regions of whole genomes will help to better understand the evolution and adaptation of closely related microbes and E. coli in particular.

Keywords: Phylogenomics, Collinearity, Pathogenicity, Escherichia coli, Adaptation

\footnotetext{
* Correspondence: linkui@bnu.edu.cn

College of Life Sciences, Beijing Normal University, No 19 Xinjiekouwai Street, Beijing 100875, China
}

\section{Biomed Central}

(c) 2012 Zhang and Lin; licensee BioMed Central Ltd. This is an Open Access article distributed under the terms of the Creative Commons Attribution License (http://creativecommons.org/licenses/by/2.0), which permits unrestricted use, distribution, and reproduction in any medium, provided the original work is properly cited. 


\section{Background}

Escherichia coli is one of the most important model organisms in both biology and medicine. Many major findings have emerged from the study of $E$. coli, including bacterial conjugation, recombination and genetic regulation. More importantly, E. coli plays important roles in the intestinal tract of humans and other vertebrates, especially in the lower section. There are more than a billion E. coli cells in the intestines of a healthy human [1]. Unfortunately, several $E$. coli strains can cause intestinal and extraintestinal diseases, such as diarrhea, urinary tract infection, septicemia, pneumonia and meningitis, in humans and animals [2]. The availability of an increasing number of complete E. coli genomes has revealed that E. coli exhibits high diversity at the whole-genome level. Comparative genomic analyses have demonstrated that the diversity among natural isolates of $E$. coli is extraordinarily high, and the average genome-wide conservation across different strains is less than $50 \%$ [1]. Therefore, $E$. coli is an ideal candidate for studying how the relationship between a bacterium and its host can fluctuate between commensalism and pathogenicity [3].

In general, at the whole-genome level, two main categories of methods are used to assess phylogenetic relationships among prokaryotes (i.e., phylogenomic analysis). One method is based on the concept of orthology, in which sequence alignment is the core computational method. Many approaches, such as gene content, gene order, multilocus sequence typing (MLST) and supertree or super-matrix methods, belong to this category [1,4-6]. Another approach is based on the frequencies of $K$-mer oligonucleotides and does not employ an alignment [6,7]; this type of method emphasizes the importance of genome content and organization. Intuitively, for phylogenomic analysis, we are seeking one or a set of genomic features that can be used as indicators/markers to robustly and correctly reveal the evolutionary relationships among a group of organisms of interest. In addition, we are also interested in features that are functional units, which could act as a bridge between genomic diversity and phenotypic differences. Within bacterial systems, the concept of an operon satisfies these two criteria. Operons are groups of genes that exhibit physical clustering within the genome and are typically transcribed in a single mRNA [8]. Genes within the same operon usually have related functions, and some of these genes may be employed in the same pathway. Regulatory genes are also commonly located in close proximity to the genes that are being regulated [8]. Although certain operons may comprise genes with no clear functional relationship, these genes may be required under the same environmental conditions even though they are involved in different pathways [9]. Unfortunately, many, if not all, operons predicted in databases to date consist only of structural genes that lack expressional regulatory elements. It is well known that the correct expression of genes must remain faithful to the specific genetic background. In addition, certain relatively large clusters of genes that have related functions, but do not belong to the same operon, have been described [10]. Therefore, it is currently assumed that predicted operons may be difficult to use in practice as indicators/markers for phylogenomic studies. With the availability of an increasing number of closely related or intraspecific prokaryotic genomes, as well as the advent of whole-genome alignment algorithms $[11,12]$, there is an opportunity to implement phylogenomic analyses of the evolution and ecological adaptation of these organisms on the whole-genome scale. To this end, we chose one type of genomic feature, called locally collinear blocks (LCBs), to study the evolutionary relationships and potential ecological adaptations of $E$. coli on the whole-genome scale. In principle, LCBs from closely related organisms or within one species should contain useful phylogenomic signals regarding their evolutionary histories. Each LCB, also known as a collinear region, is a region of DNA sequence that is shared by two or more genomes that are being studied [11]. Clearly, if an LCB is sufficiently large, it is likely to contain one or more consecutive genes with related functions in addition to their regulatory regions. Therefore, LCBs that are present or absent in either genome may satisfy both of the aforementioned criteria for feasible genomic markers; if these criteria are met, the analysis of LCBs should reveal a comprehensive history of the evolutionary and ecological adaptation of E. coli genomes.

To test our hypothesis, we studied the vertical and phenetic relationships of 34 strains of $E$. coli at the level of LCBs. First, we identified potential LCBs using the Mugsy program [12]. Next, we divided the LCBs into two groups according to their occurrence among the strains: core and variable LCBs. The core LCBs are the set of collinear regions shared by all of the studied strains, whereas the variable LCBs are the set of collinear regions that were absent in at least one of the 36 strains. Then we constructed two phylogenies based on the LCBs from each of these two groups. The phylogeny based on core LCBs tends to reflect the vertical evolutionary history of the strains (i.e., the evolutionary phylogeny). In contrast, the second phylogeny, based on the variable LCBs is likely to reveal the whole-genome similarities of extant strains (i.e., the similarity phylogeny). In the evolutionary phylogeny, the strains were clustered into groups as known phylogroups. Within each phylogroup, strains were grouped according to their respective pathotypes. These patterns indicate that it is feasible to use LCBs as indicators/markers to infer intraspecific phylogenies. We also found that the B2 phylogroup 
occured at the base of the evolutionary phylogeny, thereby suggesting that the ancestor of E. coli / Shigella was an opportunistic pathogen. Such a pathogen may be harmless under certain environmental conditions and pathogenic in other settings [7]. A comparison of the evolutionary and similarity phylogenies shows that Shigella may have at least three origins. We scrutinized the common properties of Shigella that were missing in other E. coli genomes and found that the common LCBs from their genomes were mainly influenced by mobile genetic elements. This finding implies that Shigella may have experienced a convergent evolution event via horizontal gene transfer (HGT) and acquired similar phenotypes during the course of evolution. Interestingly, by inspecting specific branches of the similarity phylogeny and correlating the branch support of LCBs with key branches in the evolutionary phylogeny, we identified putative LCBs that may be relevant to the pathogenicity of certain strains. Moreover, by analyzing the annotated genes within these regions, additional details on the evidence associated with pathogenicity were revealed, which may provide clues for further experimental evaluation. We believe that such phylogenomic studies, which examine collinear regions of whole genomes, will help to better understand the evolution and adaptation of microbes and E. coli in particular.

\section{Results}

\section{Identification of the LCBs for phylogenomic analysis}

Among closely related genomes, phylogenetic information should be inferred at the whole-genome scale. To precisely reconstruct the evolutionary phylogeny of the strains studied here, we identified as many potential collinear genomic regions as possible using a tool named Mugsy. Of the identified LCBs, we observed many gaps within several collinear regions. To ensure the quality of the LCB alignment, we filtered the collinear regions using the cutoff values defined in the Methods. A smaller cutoff value corresponded to fewer allowed alignment gaps. The occurrence of LCBs was not uniform among the strains, and their distribution displayed a U-like shape. The identified collinear regions tended to be either shared by most (right portion of Figure 1) or a few strains (left portion of Figure 1). Interestingly, this pattern is identical to that observed for individual genes [1]. This may be due to the fact that the LCBs we identified are mainly composed of genes. At the operon level, it has reported that bacterial genomes usually contain a small number of highly conserved operons and a much larger number of unique or rare ones [13]. After operons form, many of them are lost through the deletion of one or more genes contained within the operon [9]. Therefore, few operons are conserved across all or even the majority of genomes. LCBs existing in only a few strains are more than that present in all or most of the strains. This pattern is similar with that of operons, indicating that the collinear regions identified here might appear to have experienced an evolutionary history similar to that of operons. After being filtered using a cutoff value of 1.01, 412 and 35 LCBs remained, which are shared by 2 and all 36 strains, respectively. These 35 core LCBs with a combined length of 62,605 bp comprise $\sim 1 \%$ of the average length of E. coli genomes (Table 1).

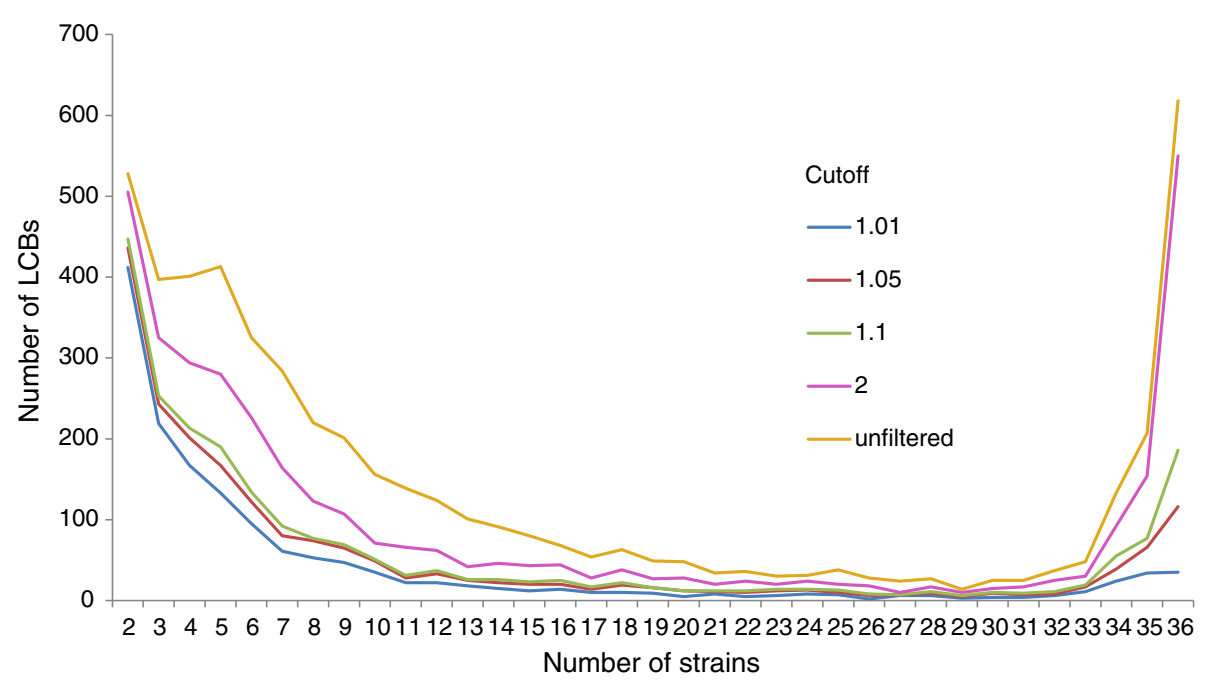

Figure 1 The distribution of the number of LCBs shared by the strains. Before being filtered, 528 and 618 LCBs were shared by 2 and 36 strains, respectively (orange). After being filtered using a cutoff of 1.01, 412 and 35 LCBs shared by 2 and 36 strains, respectively, remained (blue). A cutoff value of 1.01 means that the length of the gaps in the LCBs is less than $1 \%$ of the length of the non-gap regions. 
Table 1 The length of the 35 core LCBs

\begin{tabular}{|c|c|}
\hline$\overline{L C B}$ ID & Length (bp) \\
\hline 41 & 12,529 \\
\hline 261 & 5,326 \\
\hline 350 & 4,263 \\
\hline 366 & 4,095 \\
\hline 428 & 3,506 \\
\hline 435 & 3,457 \\
\hline 474 & 3,146 \\
\hline 527 & 2,805 \\
\hline 539 & 2,694 \\
\hline 629 & 2,169 \\
\hline 640 & 2,125 \\
\hline 678 & 1,938 \\
\hline 692 & 1,814 \\
\hline 798 & 1,388 \\
\hline 810 & 1,323 \\
\hline 879 & 1,125 \\
\hline 896 & 1,074 \\
\hline 918 & 1,009 \\
\hline 964 & 1,062 \\
\hline 974 & 898 \\
\hline 991 & 852 \\
\hline 1047 & 727 \\
\hline 1178 & 535 \\
\hline 1182 & 533 \\
\hline 1219 & 489 \\
\hline 1225 & 487 \\
\hline 1231 & 480 \\
\hline 1781 & 176 \\
\hline 1792 & 174 \\
\hline 2325 & 101 \\
\hline 2524 & 83 \\
\hline 2789 & 65 \\
\hline 2836 & 62 \\
\hline 3282 & 63 \\
\hline 3530 & 32 \\
\hline Total & 62,605 \\
\hline
\end{tabular}

Two types of phylogenies for E. coli / Shigella

After filtering using a cutoff value of 1.01, 35 and 1493 core and variable LCBs remained, respectively. Based on these two sets of LCBs, we constructed two different phylogenies using the LCBs as the phylogenomic signatures. The first phylogeny (Figure 2A), constructed using the core LCBs, is likely to reflect the lineal evolutionary history among the strains (i.e., the evolutionary phylogeny)
[7]. Based on the presence and absence of the variable LCBs, the second phylogeny (Figure 2B) is likely to reveal the similarities among the extant strains (i.e., the similarity phylogeny) [7], especially if the strains experienced convergent or divergent evolution. In the evolutionary phylogeny constructed here, the strains were clustered into groups according to their phylogroups, and within each phylogroup, the strains were clustered corresponding to their pathotypes (commensal, ExPEc or InPEc). These results indicate that core LCBs are good markers of intraspecific relationships. In our similarity phylogeny, however, most strains were not grouped with similar phylogroups or pathotypes.

\section{The evolution of Shigella}

Shigella was once elevated to the genus status based on its ability to cause a specific type of diarrhea. However, from an evolutionary perspective, all Shigella strains should be classified as E. coli [14]. In our evolutionary phylogeny, Shigella strains were divided into three clades: one clustering with B1, a second with E and a third group independent from other phylogroups. This result is in agreement with the tree of Touchon et al. [1]. In our similarity phylogeny, however, Shigella formed a monophyletic clade. This is in agreement with those trees that are likely to reveal the genome similarities $[4,7,15]$. Shigella strains scattered across three groups in the evolutionary phylogeny, while they formed a monophyletic clade in the similarity phylogeny. This pattern suggests that there were multiple origins of Shigella, which is in accordance with the findings of Rolland et al. [16], Haggerty et al. [17] and Pupo et al. [14]. However, these results are inconsistent with the theory of Escobar-Paramo et al. [18], who suggested that there was a single origin of Shigella. Interestingly, we also observed that most of the genes along the collinear regions specific to these six Shigella genomes correspond to transposases, insertion sequences or antigens (Additional file 1: Table S1). This observation may suggest that distantly related Shigella strains probably acquired a specific set of genes related to their extant phenotype and underwent convergent evolution. In addition, Shigella strains also achieved fitness through the inactivation or loss of genes incompatible with the virulence, which could occur by IS mobilization [19].

\section{Putative genomic signatures related to pathogenicity}

There are three types of factors that may be associated with the pathogenicity of $E$. coli. First, pathogenic strains are considered to differ from non-pathogenic strains primarily by the arrival of virulence factors (VFs) through HGT events $[20,21]$. Nonetheless, the integration, retention and expression of new incoming genes cannot deviate from the specific genetic background [22]. Second, 


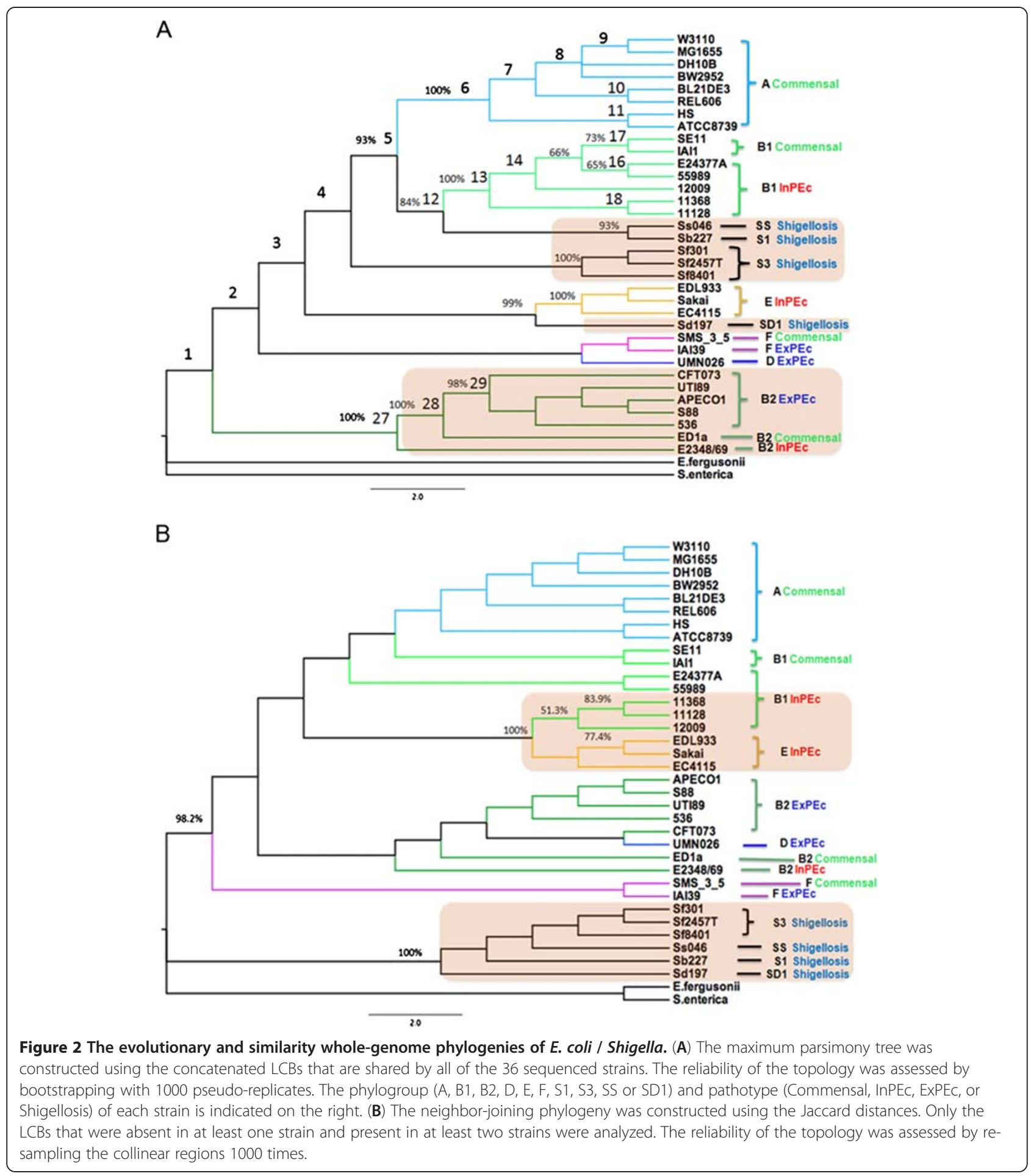

commensal E. coli can modify their genomes through deletions, point mutations or other DNA rearrangements to adapt to specific environments and cause disease in a host [20]. Third, certain types of virulence are coincidental byproducts of commensalism [23].

In the evolutionary phylogeny (Figure 2A), we observed that the seven B2 strains (CFT073, UTI89, 536,
APEC O1, S88, ED1 and O127:H6 E2348/69) clustered together according to their pathotypes, and the corresponding bootstrap values were extraordinarily high. Moreover, the five extraintestinal pathogenic E. coli strains clustered together, and within the phylogroup B2, they were the most derived subgroup. These seven B2 strains originated from the same progenitor but had 
different pathotypes. Therefore, we assume that there are certain variations in their DNA regions that may be related to their pathogenicity. To this end, we examined those LCBs that strongly support node 29 of the evolutionary phylogeny but conflict with node 28 (bottom portion of Figure 2A). Strikingly, only one collinear region (1047) was found in our filtered dataset (Table 2), and this region contained one annotated gene named $y d j M$. The product of the gene $y d j M$ is an inner membrane protein regulated by LexA and is a member of the SOS network. It has been reported that the SOS response is related to the evolution and dissemination of antibiotic resistance as well as the synthesis and dissemination of virulence [24]. We performed a multiple sequence alignment of the seven DNA segments of the LCB 1047 using the CLUSTALW program [25] with the default settings. We found that almost all of the nucleotide substitutions within the genes were synonymous (Figure 3). However, within the 5'-region of the gene, we found one nucleotide substitution ( $\mathrm{T}$ to $\mathrm{C}$ ). This mutation may be closely related to the pathogenicity of the pathogenic strains because the substitution occurs within the LexA binding sites [26]; these results indicate that it is worth performing an experimental evaluation in the future.

In the similarity phylogeny (Figure 2B), we observed that three EHEC E subgroups and three EHEC B1 subgroups clustered together, and the corresponding bootstrap value was $100 \%$. These six EHEC strains originated from different progenitors and belong to the same pathotype; therefore, we assume that they may have obtained specific regions related to their pathogenicity through HGT. To test this hypothesis, we examined the LCBs that are only present in these six strains and found that 37 collinear regions satisfied this criterion. Of these LCBs, only 17 have gene annotations. Although most of the gene products are annotated as 'hypothetical' or 'putative,' two genes of phage origin and three type III secretion system (T3SS) effector genes (i.e., espL2, nleB1 and $n l e E$ ) were also found (Additional file 2: Table S2). Nadler et al. [27] found that NleE and NleB play important roles in the interplay between host and pathogen. NleE is sufficient to inhibit NF-kB signaling, which leads to the eradication of the pathogen. In addition, NleB can enhance NleE activity. We performed a BLAST [28] search of the LCBs that contain these three genes against the other 30 genomes under study. Except for strain E2348/69, which belongs to the EPEC B2 phylogroup, no similar genes were found within the genomes of the other 29 strains, including the two outgroups. Therefore, we speculate that LCB 938, which contains the three aforementioned effector proteincoding genes, may be associated with the common pathotypes of these six strains. In addition, we believe
Table 2 The values of the partitioned branch support (PBS) of Node28 and Node29

\begin{tabular}{|c|c|c|}
\hline \multirow{2}{*}{$\begin{array}{l}\text { LCB } \\
\text { ID }\end{array}$} & \multicolumn{2}{|c|}{ PBS } \\
\hline & Node28 & Node29 \\
\hline 527 & 12 & 27 \\
\hline $1047^{a}$ & -1 & 4 \\
\hline 435 & 2 & 4 \\
\hline 428 & 1 & 2 \\
\hline 539 & 4 & 2 \\
\hline 350 & -7 & 0 \\
\hline 918 & -5 & 0 \\
\hline 2325 & -3 & 0 \\
\hline 2524 & -1 & 0 \\
\hline 1182 & -1 & 0 \\
\hline 896 & -1 & 0 \\
\hline 3530 & 0 & 0 \\
\hline 3282 & 0 & 0 \\
\hline 964 & 0 & 0 \\
\hline 1231 & 0 & 0 \\
\hline 991 & 0 & 0 \\
\hline 1178 & 0 & 0 \\
\hline 2789 & 0 & 0 \\
\hline 1781 & 0 & 0 \\
\hline 1792 & 0 & 0 \\
\hline 1219 & 1 & 0 \\
\hline 798 & 2 & 0 \\
\hline 474 & 4 & 0 \\
\hline 879 & 5 & 0 \\
\hline 640 & 21 & 0 \\
\hline 629 & 21 & 0 \\
\hline 1225 & -5 & -1 \\
\hline 678 & 2 & -1 \\
\hline 974 & 2 & -1 \\
\hline 261 & 3 & -1 \\
\hline 810 & 4 & -1 \\
\hline 692 & -7 & -3 \\
\hline 41 & 30 & -5 \\
\hline 366 & 1 & -10 \\
\hline $2836^{\mathrm{b}}$ & - & - \\
\hline
\end{tabular}

The 35 LCBs are sorted by their PBS values for node 29 in descending order, then for node 28 in ascending order.

${ }^{a}$ This is the LCB that we are interested in. It supports node 29 while conflicts with node 28.

${ }^{\text {b}}$ The seven DNA segments of the LCB 2836 are exactly the same, therefore no value exists.

that the other 36 collinear regions may also potentially be associated with pathogenicity. Specifically, the LCB 938 could be used as a proxy for the detection of EHEC/ EPEC E. coli strains. 


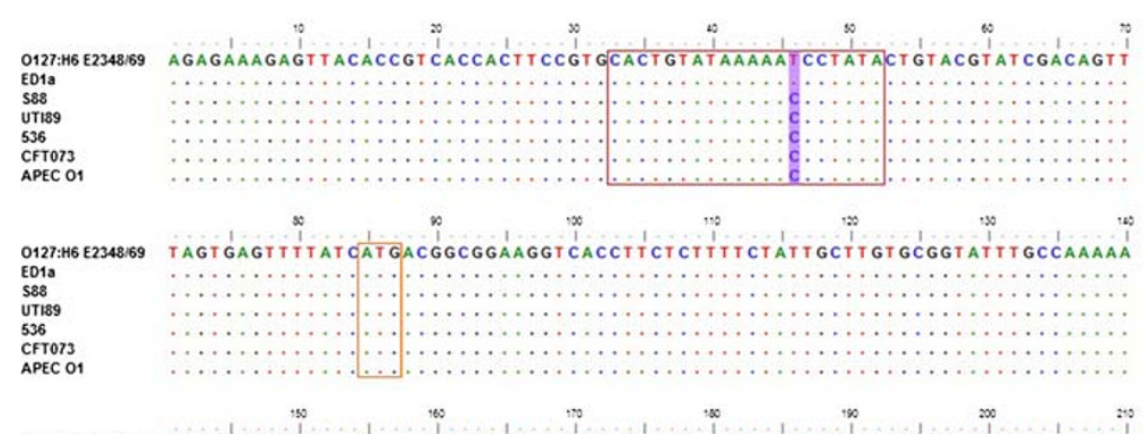

0127:H6 E2348.69 TGCCGAGCTGACGCCCGTGCTGGCACAGGGTGACTGGTGGCATATTGTCCCTTCCGCAATCCTGACGTGT ED1a

UT189

536

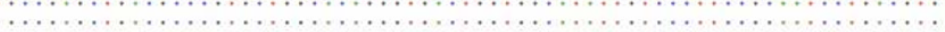

APEC 01

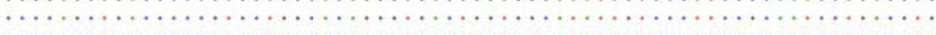

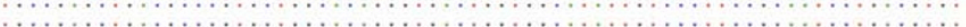

0127:H6 E2348:69 TTGTTACCGGACATCGATCACCCAAAGTCGTTTCTTGGGCAGCGATTAAAGTGGATATCAAAACCGATCG O127:H6
EO1a
S88

UT189

CFT073

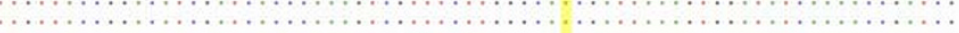
……… APEC 01

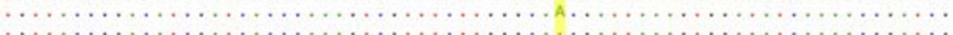

0127:H6E2348:69 CCCGCGCTTTTGGGCATCGTGGTTTTACCCACAGTCTGCTGGCGGTATTTGCGCTGCTGGCAACCTTTTA ED1a
588

UT189

536

APEC O1

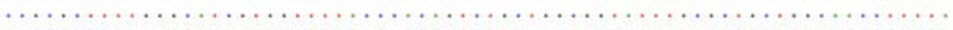

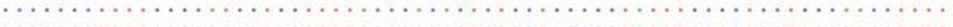
.

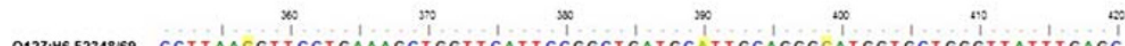
0127:H6 E2348.69 CCTTAAGGTTCCTGAAAGCTGGTTCATTCCGGCTGATGCATTGCAGGGCATGGTGCTGGGTTATTTGAGC ED1s
S88

UT189

536

APEC 01

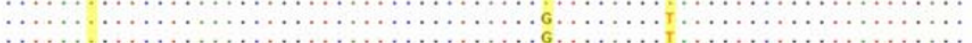

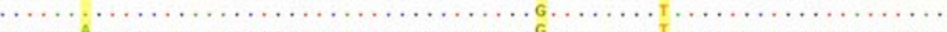

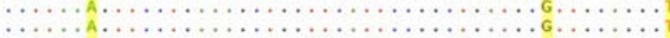
.

0127:H6 E2348:69 CACATACTTGCCGATATGCTGACACCCGCCGGTGTTCCCCTGCTCTGGCCATGCCGCTGGCGTTTCCGCT ED1a

UTi89

536

APEC 01

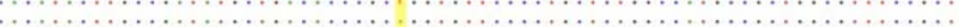

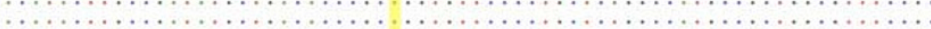
2.

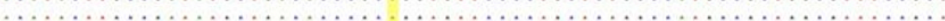

0127:H6 E2348.69 TGCCTATCCTGGTTCCCCAAAAGGGCAACCAACTGGAACGTTTAATCTGCATGGCATTATTTGTCTGGTC

UTI89

536
CFTO73
APEC O1

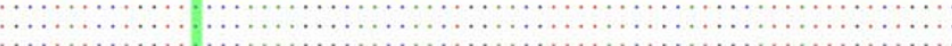

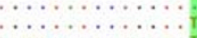
.

30

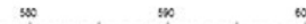

0127:H6 E2348:69 GGTATGGATGCCCCATTCATTACCCGAAAACAGCGCTGTTCGTTGGTCATCGCAAATGATCAATACCTTG ED1a

UTI8

UT189

536
CFT073
APEC 01

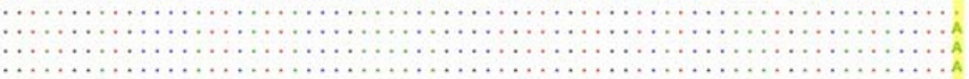

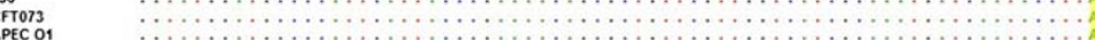

0127:H6 E2348.69 CAGATCCAGTTTCATCGACTTATTAAGCATCAGGTTGAATACTAAAAAGGCAAAAATC ICCTTTCTGGAA

ED1
S88
UTB
536

UTI89

536
CFT073
APEC 01

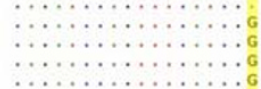

G.

M......

0127:H6 E2348/69 TAAGCAATTCCATTTGAATAT A

ED1a

UTI89

536

APEC 01

.

(n)

.

Figure $\mathbf{3}$ (See legend on next page.) 
(See figure on previous page.)

Figure 3 Substitution variants revealed by the multiple sequence alignment of the seven DNA segments (label 1047). The red inset highlights the LexA binding site of $y d j M$, and the orange inset indicates the start and stop codons of $y d j M$. Yellow and green shading highlight the synonymous and non-synonymous nucleotide substitutions, respectively, within ydjM; red shading highlights nucleotide substitutions within the $3^{\prime}$-region of $y d j M$; and blue shading shows nucleotide substitutions within the LexA binding site of ydjM.

\section{Discussion}

With the advent of high-throughput DNA sequencing technologies, an increasing number of complete genomes and microbial genomes in particular, have been sequenced in the past ten years. The development of methods for the efficient use of these valuable data in the study of evolutionary biology presents a challenge to biologists. Within each whole genome, there are many hidden features that depict the evolutionary history of the species. These features provide us with a window to the evolutionary history of life on our planet through which comprehensive phylogenetic relationships can be reconstructed [6]. In addition to the two aforementioned major categories of reconstruction methods, other evolutionary markers within complete genomes have also been used. For example, various types of rare genomic changes, such as insertions and deletions (indels), intron positions and overlapping genes, have been used to address specific phylogenetic questions [6,29]. Recently, an approach based on metabolic pathway reaction content was proposed [15]. This method considers the effect of the metabolic networks on phenotypic behavior. In our present work, we used information regarding collinear genomic regions to assess two types of relationships, i.e., evolutionary and phenetic, among 34 E. coli strains. Our results suggest that LCBs are suitable for the phylogenomic analysis of the evolutionary relationships among closely related genomes. More interestingly, LCBs could, to a certain extent, reflect the phenetic relationships of the genomes in which they reside.

To date, several tools for multiple alignments of whole genomes at the DNA scale have been proposed $[11,12]$. In this work, we identified a total of 5097 LCBs using the Mugsy program. Figure 4 shows the number and length distributions of LCBs shared by different numbers of strains. Curiously, before being filtered, the number of longer LCBs (>1 kbp) shared by the majority of strains ( $>=34$ genomes) was greater than that shared by the minority of strains ( $<=4$ genomes), with the opposite trend observed for the shorter LCBs (Figure 4A). Intuitively, the LCBs shared by the minority of strains should be longer than those shared by the majority of strains. Further examination revealed the existence of many gaps in several LCBs, especially in the longer LCBs. Because even small genomic variations may occasionally change the functions of genes, we filtered the original 5097 LCBs using a strict cutoff value (1.01), which means the length of gaps in each LCB is smaller than 1\% of the length of the non-gap regions. After filtering, only $78 \%$ and $6 \%$ of the original LCBs shared by 2 and 36 strains, respectively, remained (Figure 1). The number of LCBs shared by two strains decreased from 528 to 412, and the number of LCBs shared by all 36 strains decreased from 618 to 35 . Moreover, after being filtered, the number of LCBs shared by the majority of strains was less than that shared by the minority of strains irrespective of the length of each LCB (Figure 4B). This observation suggests that longer LCBs tend to inherit genetic materials from their vertical ancestors rather than by HGT. Based on these data, together with the patterns depicted in Figures 4A and B, we conclude that the longer LCBs should have experienced many insertions or deletions during the evolution of the strains.

Although many methods have been designed for the reconstruction of phylogenetic trees among species, many of them are not suitable for inferring intraspecific phylogenetic relationships. Lukjancenko et al. reported that neither $16 \mathrm{~S}$ rRNA sequences nor MLST gene sets are suitable for the analysis of inter-strain relationships within a species or between closely related species [5]. However, analyses based on whole-genome signatures appear to work well for inter-strain comparisons and the study of closely related genomes. Touchon et al. [1], Ogura et al. [4] and Lukjancenko et al. [5] found that gene-alignment-based methods could group $E$. coli strains in a meaningful way. Touchon et al. built a phylogeny based on 1,878 core genes. Ogura et al. built two phylogenies based on 345 orthologous CDS and a gene repertoire containing 12,940 CDS. Lukjancenko et al. performed a clustering of $E$. coli and related species based on their variable gene content. In a study based on alignment-free inference, Sims et al. [7] discovered that the feature frequency profile (FFP) method could also provide useful information for comparing the whole-genome sequences of $E$. coli. They constructed two phylogenies using the frequency vectors of oligonucleotides with a length of $24 \mathrm{nt}$. In general, all previously inferred phylogenies can be separated into two categories: those based on the core genes or features, which are similar to our evolutionary phylogeny, and those based on the variable gene content or other genomic features or metabolic pathways, which are similar to our similarity phylogeny. Our evolutionary phylogeny is, overall, congruent with those constructed using similar methods 

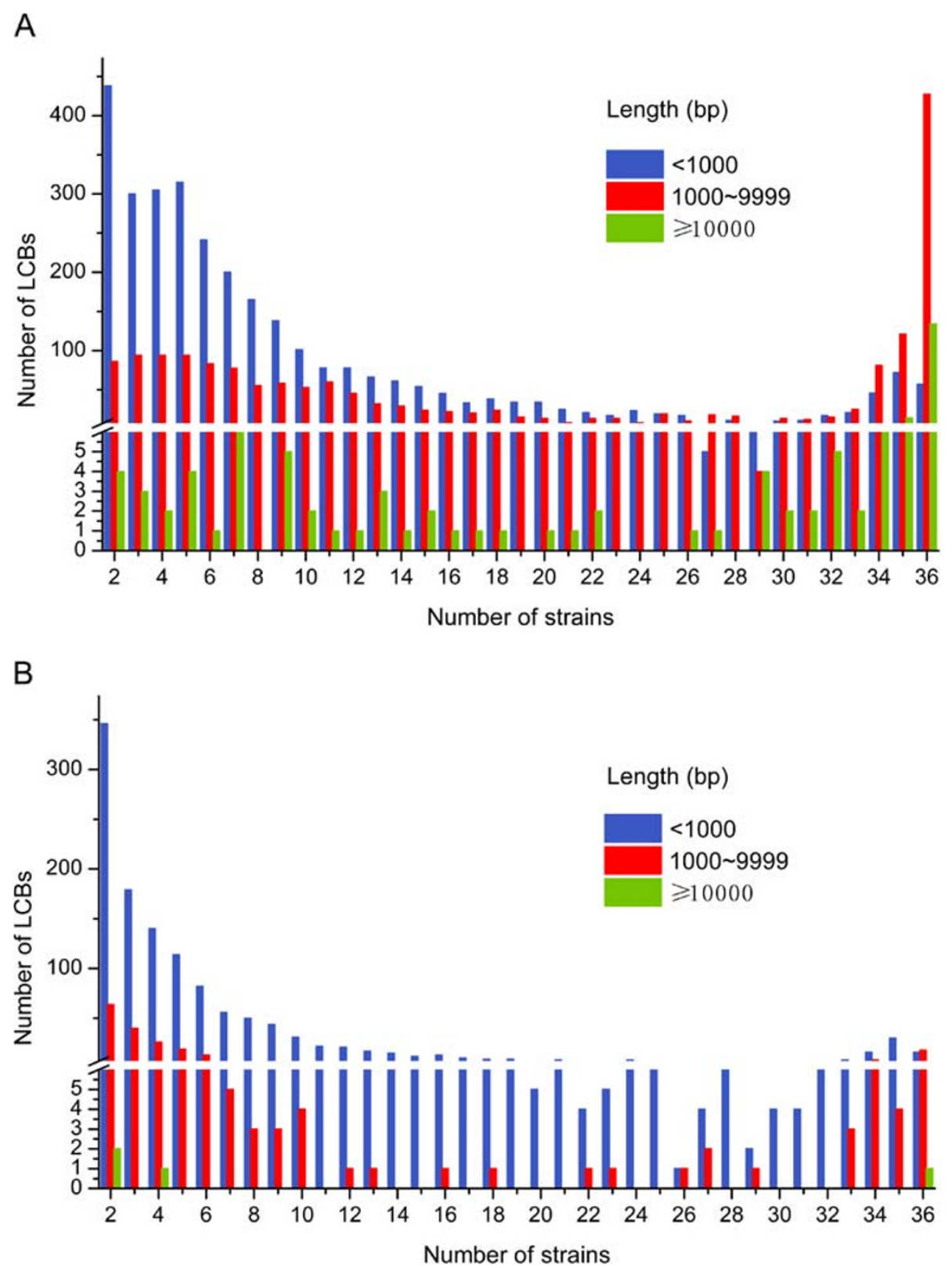

Figure 4 The number and length distributions of the LCBs. (A) Distributions of the unfiltered LCBs. (B) Distributions of the filtered LCBs with a cutoff value of 1.01

where B2 is the basal phylogroup. In contrast, the Shigella strains in our similarity phylogeny were distinctly distant from the other strains. This pattern is in agreement with several previous studies $[5,15]$, whereas there are certain differences relative to other studies [7]. Regarding the methodology, we believe that the comparison using K-mer frequency vectors is similar to the LCB-based comparison used here. The analysis of $K$-mer features, which are good markers for inferring evolutionary histories of organisms across both large and small evolutionary distances and regardless of the genome size variation, may appear to behave differently from other methods when depicting phenotypic behaviors because $K$-mer features contain insufficient functional information. Nonetheless, LCBs, which tend to be conserved between closely related species, could provide more valuable evolutionary and functional information for the analysis of both evolutionary histories and phenotypic behaviors of closely related species and intraspecific genomes in particular. Conversely, because there is minimal conservation of LCBs at great evolutionary distances, it is difficult to provide sufficient information for phylogenomic analyses of distantly related species [30,31]. 


\section{Conclusions}

Our results demonstrate that collinear regions are suitable for analyzing both the evolutionary history and ecological adaption of E. coli. For closely related genomes, collinear regions (LCBs in this study) at the DNA level are reliable genomic features used for phylogenomic analysis while linking the genomic diversity with phenotypic differences in a meaningful way. Our evolutionary phylogeny based on common collinear regions reveals potential signatures exhibiting both meaningful phylogenetic and phenotypic patterns. A comparison of the evolutionary and similarity phylogenies suggests that Shigella experienced a convergent evolution event. This group originated from at least three distinct progenitors and evolved into one phenetically similar group. By inspecting certain interesting branches of the evolutionary and similarity phylogenies, we found that the pathogenicity of a strain may be associated with both the arrival of virulence factors through HGT and the modification of genomes via mutations. More interestingly, specific collinear regions could be used as proxies for the detection of certain subgroups of $E$. coli. Given these findings, future experimental validations are needed to confirm the correlation between collinear regions and the pathogenicity inferred in this study.

\section{Methods}

\section{Bacterial genomes and gene annotations}

The genome sequences, which were previously manually re-annotated by experts, were downloaded from the MicroScope database [32] in June 2011. Thirty-four strains of E. coli, including $28 \mathrm{E}$. coli strains and six Shigella strains that belong to the E. coli species [14], as well as the two outgroups ATCC_35469 ${ }^{\mathrm{T}}$ and Typhimurium LT2 from E. fergusonii and S. enterica, respectively, were used in this study (Table 3). This set of strains covers all of the main phylogenetic groups (A, B1, B2, D, $\mathrm{E}$ and F) [33] and various pathogenic behaviors (commensal, ExPEc and InPEc) of E. coli strains as well as four phylogenetic groups (S1, S3, SD1 and SS) [14] of Shigella strains (the pathotype of which are marked as Shigellosis). The phylogenetic groups and pathotypes of these strains were obtained from previous publications $[7,15]$ and the GOLD database [34].

Multiple whole-genome alignments of the strain genomes Whole-genome comparisons were performed using the Mugsy program version 1.2.2, which is computationally efficient and can effectively align closely related whole genomes compared to other tools [12]. All chromosomes from the 36 genomes were aligned using the parameters "-distance $=1000$ " and "-minlength $=30$ ", which specify the maximum genomic distance between adjacent anchors and the minimum block length, respectively. To guarantee the quality of the Mugsy output file, a list of LCBs was further filtered using a cutoff value (score $\leq$ 1.01). This value was defined as follows:

$$
\text { score }=\frac{L_{c} \times n}{\sum_{i}^{n} l_{i}}
$$

Where $L_{c}$ is the length of the LCB (including the gap), $n$ is the number of strains in which this LCB is present and $l_{i}$ is the length of the LCB from strain $i$ (excluding gaps). When an LCB was contained within another LCB, the internal LCB was discarded and the longer LCB was retained. This set of filtered LCBs was then used to perform the phylogenomic analysis.

\section{Phylogenies based on LCBs}

Two tree-construction methods were used: $(i)$ an evolutionary phylogeny based on core LCBs that are shared by all the studied genomes and (ii) a similarity phylogeny based on variable LCBs that are absent in at least one genome.

Evolutionary phylogeny The LCBs shared by all of the studied genomes were assembled into a concatenated LCB matrix in NEXUS format using the ASAP program [35]. A maximum parsimony phylogenomic tree was then constructed using PAUP* $4 \mathrm{~b} 10$ [36] using the heuristic search option with 200 random taxon additions and TBR branch swapping. The partitioned branch supports (PBSs) [37], which identify the relative contribution of each of the data partitions to the concatenated tree at each node, were also calculated using ASAP. Positive, negative and zero PBS values signify character support, conflict and neither support nor conflict, respectively.

Similarity phylogeny The similarity phylogeny was constructed by the neighbor joining methods using the PHYLIP package version 3.68 [38]. Briefly, a matrix of LCBs was constructed with values of 1 or 0 where each row corresponds to an LCB and each column represents a genome. A cell $(i, j)$ in the matrix is equal to 1 if $\mathrm{LCB}$ $i$ is present in genome $j$ or equal to 0 if the LCB is absent. The Jaccard distances, defined below, were calculated and used to generate the distance matrix for the phylogenetic inference. The LCBs present in only one genome or in all of the genomes were not included in this analysis. Bootstrap values were computed for each inner node by re-sampling the rows of the matrix 1000 times.

$$
J_{A, B}=\frac{M_{10}+M_{01}}{M_{10}+M_{01}+M_{11}}
$$

In the above equation, $M_{10}$ represents the number of LCBs that are present in genome $A$ but absent from 
Table 3 Main characteristics of the strains used in this study

\begin{tabular}{|c|c|c|c|c|}
\hline Strain & Phylogenetic group & Pathotype $^{a}$ & Genome size (Mb) & Accession number (MicroScope) \\
\hline \multicolumn{5}{|l|}{ Escherichia coli } \\
\hline K12 W3110 & A & Commensal & 4.5 & GBKW3110_AC_000091 \\
\hline HS & A & Commensal & 4.5 & EcHS_A NC_009800 \\
\hline ATCC8739 & A & Commensal & 4.6 & EcolC_NC_010468 \\
\hline $\mathrm{K} 12 \mathrm{DH} 10 \mathrm{~B}$ & A & Commensal & 4.6 & ECDH10B_NC_010473 \\
\hline K12 BW2952 & A & Commensal & 4.5 & BWG_NC_012759 \\
\hline BL21(DE3) & A & Commensal & 4.5 & ECBD_NC_012947 \\
\hline B REL606 & A & Commensal & 4.5 & ECB_NC_012967 \\
\hline K12 MG1655 & A & Commensal & 4.5 & U00096 \\
\hline E24377A & B1 & InPEc(ETEC) & 4.9 & EcE24377A_NC_009801 \\
\hline SE11 & B1 & Commensal & 4.8 & ECSE_NC_011415 \\
\hline |Al1 & B1 & Commensal & 4.6 & ECIAl1_EIAl1v2 \\
\hline 55989 & B1 & InPEC(EAEC) & 5 & EC55989_EC55v2 \\
\hline O103:H2 12009 & B1 & $\ln P E c(E H E C)$ & 5.3 & ECO103_NC_013353 \\
\hline O26:H11 11368 & B1 & $\ln P E C(E H E C)$ & 5.6 & ECO26_NC_013361 \\
\hline O111:H- 11128 & B1 & $\ln P E c(E H E C)$ & 5.2 & ECO111_NC_013364 \\
\hline CFT073 & B2 & ExPEC & 5.1 & c NC_004431 \\
\hline UTI89 & B2 & ExPEC & 5 & UTI89_C NC_007946 \\
\hline 536 & B2 & ExPEC & 4.8 & ECP_NC_008253 \\
\hline APEC 01 & B2 & ExPEc & 5 & APECO1_NC_008563 \\
\hline O127:H6 E2348/69 & B2 & $\ln P E c(E P E C)$ & 4.9 & E2348C_NC_011601 \\
\hline S88 & B2 & ExPEc & 4.9 & ECS88_ECOS88V2 \\
\hline ED1a & B2 & Commensal & 5.1 & ECED1_ED1av2 \\
\hline UMN026 & $\mathrm{D}$ & ExPEC & 5.1 & ECUMNv2_ESCUMv2 \\
\hline O157:H7 EDL933 & $E$ & $\ln P E C(E H E C)$ & 5.4 & Z NC_002655 \\
\hline O157:H7 Sakai & $E$ & $\ln P E c(E H E C)$ & 5.4 & Ecs NC_002695 \\
\hline O157:H7 EC4115 & $E$ & $\ln P E c(E H E C)$ & 5.4 & ECH74115_NC_011353 \\
\hline SMS-3-5 & $\mathrm{F}$ & Commensal & 5 & EcSMS35_NC_010498 \\
\hline IAl39 & $\mathrm{F}$ & ExPEC & 5 & ECIAI39_EIAI39v2 \\
\hline \multicolumn{5}{|l|}{ Shigella } \\
\hline S. boydii 4227 (Sb 227) & $\mathrm{S}(\mathrm{S} 1)$ & Shigellosis & 4.4 & SBO NC_007613 \\
\hline S. flexneri 2a 301 (Sf 301) & $\mathrm{S}(\mathrm{S} 3)$ & Shigellosis & 4.5 & SF NC_004337 \\
\hline S. flexneri 2a 2457 T (Sf 2457 T) & $S(S 3)$ & Shigellosis & 4.5 & S NC_004741 \\
\hline S. flexneri 58401 (Sf 8401) & $S(S 3)$ & Shigellosis & 4.5 & SFV_NC_008258 \\
\hline S. dysenteriae 1197 (Sd 197) & $\mathrm{S}(\mathrm{SD} 1)$ & Shigellosis & 4.3 & SDY NC_007606 \\
\hline S. sonnei 046 (Ss 046) & $\mathrm{S}(\mathrm{SS})$ & Shigellosis & 4.7 & SSO_NC_007384 \\
\hline \multicolumn{5}{|l|}{ Outgroup } \\
\hline E. fergusonii ATCC 35496 & Outgroup & Commensal & 4.5 & EFER_EFERv2 \\
\hline S. enterica enterica LT2 & Outgroup & Pathogen & 4.7 & STM NC_003197 \\
\hline
\end{tabular}

${ }^{a} I n P E C:$ Intraintestinal pathogenic E. coli, ExPEc: Extraintestinal pathogenic E. coli.

ETEC: enterotoxigenic E. coli, EAEC: enteroaggregative E. coli, EHEC: enterohemorrhagic E. coli, EPEC: enteropathogenic E. coli.

genome $B . M_{01}$ represents the number of LCBs that are absent from genome $A$ but present in genome $B$. Finally, $M_{11}$ represents the number of LCBs that are present in both genomes $A$ and $B$.

\section{Additional files}

Additional file 1: Table S1. List of genes along the LCBs which are specific to six Shigella strains. 
Additional file 2: Table S2. List of genes within the LCBs that are specific to six enterohemorrhagic E. coli.

\section{Abbreviations}

MLST: Multilocus sequence typing; LCB: Locally collinear block; HGT: Horizontal gene transfer; VF: Virulence factor; T3SS: Type III secretion system; FFP: Feature frequency profile; PBS: Partitioned branch support; InPEc: Intraintestinal pathogenic Escherichia coli; ExPEc: Extraintestinal pathogenic Escherichia coli; ETEC: Enterotoxigenic Escherichia coli; EAEC: Enteroaggregative Escherichia coli; EHEC: Enterohemorrhagic Escherichia coli; EPEC: Enteropathogenic Escherichia coli.

\section{Competing interests}

The authors declare that they have no competing interests.

\section{Authors' contributions}

$\mathrm{KL}$ designed the work and helped to draft the manuscript. YZ performed the work and wrote the manuscript. All authors read and approved the final manuscript.

\section{Acknowledgements}

We are grateful to the two anonymous reviewers whose comments helped us to improve the manuscript. This work was supported by the National Natural Science Foundation of China (NSFC), Grant 31121003.

Received: 20 May 2012 Accepted: 28 August 2012

Published: 7 September 2012

\section{References}

1. Touchon M, Hoede C, Tenaillon O, Barbe V, Baeriswyl S, Bidet P, Bingen E, Bonacorsi S, Bouchier C, Bouvet O, et al: Organised genome dynamics in the Escherichia coli species results in highly diverse adaptive paths. PLoS Genet 2009, 5(1):e1000344

2. Donnenberg MS: Escherichia coli: virulence mechanisms of a versatile pathogen. Academic Press: Elsevier Science; 2002.

3. Tenaillon O, Skurnik D, Picard B, Denamur E: The population genetics of commensal Escherichia coli. Nat Rev Microbiol 2010, 8:207-217.

4. Ogura Y, Ooka T, Iguchi A, Toh H, Asadulghani M, Oshima K, Kodama T, Abe H, Nakayama K, Kurokawa K: Comparative genomics reveal the mechanism of the parallel evolution of $\mathrm{O} 157$ and non-0157 enterohemorrhagic Escherichia coli. Proc Natl Acad Sci USA 2009, 106(42):17939.

5. Lukjancenko O, Wassenaar TM, Ussery DW: Comparison of 61 Sequenced Escherichia coli Genomes. Microb Ecol 2010, 60(4):708-720.

6. Delsuc F, Brinkmann H, Philippe H: Phylogenomics and the reconstruction of the tree of life. Nat Rev Genet 2005, 6(5):361-375.

7. Sims GE, Kim S-H: Whole-genome phylogeny of Escherichia coli/Shigella group by feature frequency profiles (FFPs). Proc Natl Acad Sci USA 2011, 108(20):8329-8334

8. Osbourn AE, Field B: Operons. Cell Mol Life Sci 2009, 66(23):3755-3775.

9. Price MN, Arkin AP, Alm EJ: The Life-Cycle of Operons. PLoS Genet 2006, 2(6):e96.

10. Igarashi N, Harada J, Nagashima S, Matsuura K, Shimada K, Nagashima KV: Horizontal Transfer of the Photosynthesis Gene Cluster and Operon Rearrangement in Purple Bacteria. J Mol Evol 2001, 52:333-341

11. Darling ACE: Mauve: Multiple Alignment of Conserved Genomic Sequence With Rearrangements. Genome Res 2004, 14(7):1394-1403.

12. Angiuoli SV, Salzberg SL: Mugsy: fast multiple alignment of closely related whole genomes. Bioinformatics 2011, 27:334-342.

13. Koonin EV: Evolution of genome architecture. Int J Biochem Cell Biol 2009, 41(2):298-306

14. Pupo GM, Lan R, Reeves PR: Multiple independent origins of Shigella clones of Escherichia coli and convergent evolution of many of their characteristics. Proc Natl Acad Sci USA 2000, 97:10567-10572.

15. Vieira G, Sabarly V, Bourquignon P-Y, Durot M, Le Fevre F, Mornico D, Vallenet D, Bouvet O, Denamur E, Schachter V, et al: Core and Panmetabolism in Escherichia coli. J Bacteriol 2011, 193(6):1461-1472.

16. Rolland K, Lambert-Zechovsky N, Picard B, Denamur E: Shigella and enteroinvasive Escherichia coli strains are derived from distinct ancestral strains of E. coli. Microbiology 1998, 144:2667-2672.
17. Haggerty LS, Martin FJ, Fitzpatrick DA, Mclnerney JO: Gene and genome trees conflict at many levels. Phil Trans R Soc B 2009, 364:2209-2219.

18. Escobar-Paramo P, Giudicelli C, Parsot C, Denamur E: The evolutionary history of Shigella and enteroinvasive Escherichia coli revised. J Mol Evol 2003, 57:140-148.

19. Maurelli AT: Black holes, antivirulence genes, and gene inactivation in the evolution of bacterial pathogens. FEMS Microbiol Lett 2007, 267(1):1-8.

20. Kaper JB, Nataro JP, Mobley HLT: Pathogenic Escherichia coli. Nat Rev Microbiol 2004, 2(2):123-140.

21. Ochman H, Lawrence JG, Groisman EA: Lateral gene transfer and the nature of bacterial innovation. Nature 2000, 405:299-304.

22. Escobar-Paramo $P$, Clermont $O$, Blanc-Potard A-B, Bui H, nec àCLB, Denamur $E$ : A specific genetic background is required for acquisition and expression of virulence factors in Escherichia coli. Mol Biol Evol 2004, 21 (6):1085-1094.

23. Le Gall T, Clermont O: Gouriou Sp, Picard àB, Nassif X, Denamur E, Tenaillon O: Extraintestinal virulence is a coincidental by-product of commensalism in B2 phylogenetic group Escherichia coli strains. Mol Biol Evol 2007, 24(11):2373-2384.

24. Butala M, Žgur-Bertok D, Busby SJW: The bacterial LexA transcriptional repressor. Cell Mol Life Sci 2009, 66(1):82-93.

25. Thompson JD, Higgins DG, Gibson TJ: CLUSTAL W: improving the sensitivity of progressive multiple sequence alignment through sequence weighting, position-specific gap penalties and weight matrix choice. Nucleic Acids Res 1994, 22:4673-4680

26. Fernández De Henestrosa AR, Ogi T, Aoyagi S, Chafin D, Hayes Jل J Ohmori H, Woodgate R: Identification of additional genes belonging to the LexA regulon in Escherichia coli. Mol Microbiol 2000, 35(6):1560-1572.

27. Nadler C, Baruch K, Kobi S, Mills E, Haviv G, Farago M, Alkalay I, Bartfeld S, Meyer TF, Ben-Neriah Y, et al: The Type III Secretion Effector NleE Inhibits NF-kB Activation. PLoS Pathog 2010, 6(1):e1000743.

28. Altschul SF, Gish W, Miller W, Myers EW, Lipman DJ: Basic Local Alignment Search Tool. J Mol Biol 1990, 215(3):403-410.

29. Luo Y, Fu C, Zhang D-Y, Lin K: Overlapping genes as rare genomic markers: the phylogeny of gamma-Proteobacteria as a case study. Trends Genet 2006, 22(11):593-596.

30. Mushegian AR, Koonin EV: Gene order is not conserved in bacterial evolution. Trends Genet 1996, 12:289-290.

31. Wolf YI, Rogozin IB, Kondrashov AS, Koonin EV: Genome alignment, evolution of prokaryotic genome organization and prediction of gene function using genomic context. Genome Res 2001, 11:356-372.

32. Vallenet D, Engelen S, Mornico D, Cruveiller S, Fleury L, Lajus A, Rouy Z, Roche D, Salvignol G, Scarpelli C, et al: MicroScope: a platform for microbial genome annotation and comparative genomics. Database 2009, 2009:bap021.

33. Denamur E, Picard B, Tenaillon O: Population Genetics of Pathogenic Escherichia coli. In Bacterial Population Genetics in Infectious Disease. Edited by Robinson DA, Falush D, Feil EJ. Hoboken: John Wiley \& Sons, Inc; 2010.

34. Pagani I, Liolios K, Jansson J, Chen I-MA, Smirnova T, Nosrat B, Markowitz VM, Kyrpides NC: The Genomes OnLine Database (GOLD) v. 4: status of genomic and metagenomic projects and their associated metadata. Nucleic Acids Res 2012, 40:D571-D579.

35. Sarkar I, Egan MG, Coruzzi G, Lee EK, Desalle R: Automated simultaneous analysis phylogenetics (ASAP): an enabling tool for phlyogenomics. BMC Bioinforma 2008, 9(1):103.

36. Swofford D: PAUP*: phylogenetic analysis using parsimony, version 4.0 b10:: 2003

37. Baker RH, DeSalle R: Multiple sources of character information and the phylogeny of Hawaiian drosophilids. Syst Biol 1997, 46(4):654-673.

38. Felsenstein J: PHYLIP (Phylogeny Inference Package) version 3.6. Distributed by the author. Seattle: Department of Genome Sciences, University of Washington; 2005.

doi:10.1186/1471-2148-12-174

Cite this article as: Zhang and Lin: A phylogenomic analysis of Escherichia coli / Shigella group: implications of genomic features associated with pathogenicity and ecological adaptation. BMC Evolutionary Biology 2012 12:174. 